\title{
Antenna Measurement System Operating at W and J Millimeter Wave Bands
}

\author{
A. Muñoz-Acevedo ${ }^{a}$, M. Sierra-Castañer ${ }^{\mathrm{a}}$ J.L. Besada ${ }^{\mathrm{a}}$ \\ ${ }^{\mathrm{a}}$ Radiation Group, Technical University of Madrid, Spain
}

\begin{abstract}
A millimeter wave antenna measurement system is presented. The frequencies of operation are around $100 \mathrm{GHz}$ and $300 \mathrm{GHz}(\mathrm{W} / \mathrm{J}$ Bands). The facility is a single reflector compact range system. Design tasks deal with the serrated reflector and the corresponding millimeter wave instrumentation design.
\end{abstract}

\section{INTRODUCTION AND BACKGROUND}

A NTENNA measurements at millimeter wavelengths constitute the last part of mm-wave antenna development. An antenna measurement system is a facility whose purpose is to carry out the radiation pattern evaluation of a particular antenna prototype. More specifically, CATR systems are measurement facilities whose purpose is to generate a plane wave field distribution, which fulfills the far-field measurement conditions needed for real-time antenna characterization.

At millimeter wavelengths, main constraints in the development of an operating CATR appear in the subsequent stages of the whole process. At design stages, CAD analysis are commonly very time consuming and flexible design geometries can be, moreover, difficult to analyze. In the construction stages, mechanization tolerances become increasingly strict, while assembly requires accurate alignment tests.

Technical University of Madrid [UPM] is, in the present, working on extending the frequencies of operation of its measurement facilities. The option at $100 \mathrm{GHz}$ (W-band) consists on the incorporation of Agilent mm-Wavelength instrumentation in the Gregorian compact range facility [3]. On the other side, a brand new offset reflector CATR facility is being projected. This last one will be our point of interest in this communication.

\section{DESIGN PROCESS}

\section{A. Algorithm introduction}

The design stage involves the development of an offset-fed parabolic reflector with conformal serrated edge, as schematized in Fig. 1. The purpose of this reflector is to act as a collimator of the spherical wavefront from the feeder, generating a field distribution in the Quiet zone, as similar as possible as a plane wave. Thus, amplitude and phase ripples of the field in the Acquisition planes are indicators of the system overall performance [4,7].

A Hybrid GO-PWS [5] algorithm has been designed to carry out analysis-design tasks of this reflector. Mathematical formulation, results and validation of the algorithm can be found at $[1,2]$. The main feature of the

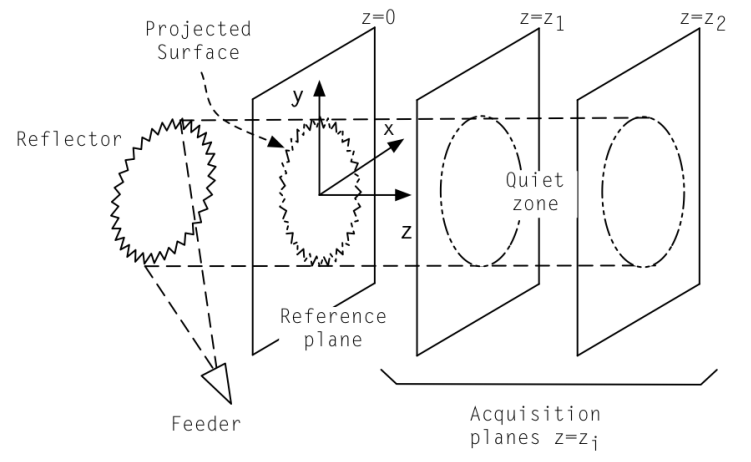

Fig. 1. Serrated edge offset reflector compact range setup
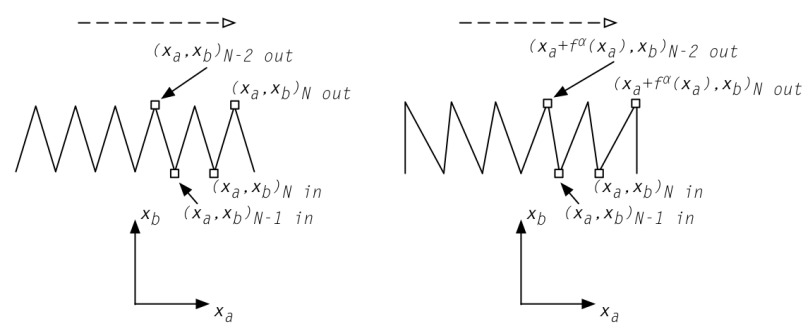

Fig. 2. Linear edge serrations distribution modulation

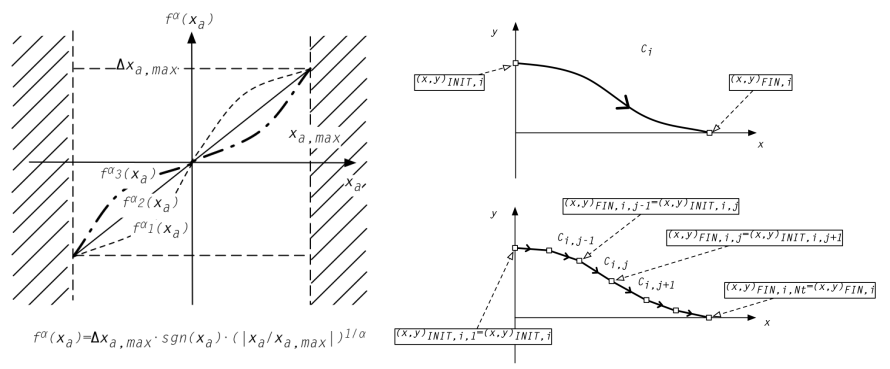

Fig. 3. Edge modulation base functions (a), cosine piecewise serrations (b)

algorithm is its analytical treatment of arbitrary-edge parabolic reflectors, in addition with accurate and computational speed. These features ensure complete and reliable design processes, maximizing the overall performance of the system from the beginning of the development.

\section{B. Geometry definition}

As mentioned before, the analysis algorithm deals with analytical treatment of the system. Thus, a finite (and small) number of parameters, arranged in a vector $\Omega=\left(\omega_{1}, \ldots, \omega_{m}\right)$ [1] is able to univocally define a particular setup. Each one of the $\omega_{i}$ parameters has its particular domain of existence, so a complete design requires a global optimization process over a bounded $\Omega$ vector. 
In this process, amplitude and phase ripples are the cost functions to be minimized. The state vector is defined by the components of $\Omega$. The choice of each one of the optimization variables attends to the relationship between the geometrical aspect that its models and the influence on electromagnetic field that wants to be achieved.

Several lines of design are carried out in the process. First of all, a square size is chosen for the serrated reflector, which maximizes the quiet zone area. Secondly, edge serrations are modulated so the diffracted rays are set away from the quiet zone [8], as described in Fig. 2. This modulation follows an analytical distribution, which is modeled through parametric functions, as shown in Fig. 3. (a). A widely-used option for serrations consists on replacing the triangular serrations by cosine-defined ones - Fig. 3. (b) -, which have been proofed to minimize the quiet zone ripple $[2,6]$. The cosine function can be, itself, modulated at different orders, which multiplies the design alternatives.

\section{RESULTS}

Amplitude as well as phase cuts of the quiet zone field in several configurations have already been shown in $[1,2]$. Here, our purpose is to show the optimum reflector design. Field is acquired $300 \mathrm{~mm}$. away from the reflector surface. The side of the reflector is $340 \mathrm{~mm}$. wide, with $80 \mathrm{~mm}$-length cosineshape serrations. The focal distance of the parabola is 500 $\mathrm{mm}$. Vectorial data of the quiet zone is handled, so rms figures of both amplitude and phase field acquisitions are available.

Rough analysis of quiet zone performance states that amplitude and phase ripple have a common trend evolution when varying geometrical configurations of the collimator. However, a finer examination reveals that amplitude and phase ripple evolution have local divergent evolutions for particular sets of reflector alternatives. It is necessary, thus, to weight phase and amplitude rms contributions separately to obtain a requirements accomplishment joint figure. The final design will be a compromise solution between amplitude performance and phase flatness.

Implementations of the different alternatives are depicted in Fig. 4. Amplitude and phase horizontal cuts of the design option are shown in Fig. 5.

Full quiet zone characterization consists on shifting the acquisition plane location, by moving the $\mathrm{z}_{\mathrm{i}}$ coordinate (Fig. 1) within the domain of interest. With this, a clear idea of the quiet zone spatial stability is obtained.

\section{CONCLUSION}

A short overview of Radiation Group developments in the field of antenna measurement facilities at $\mathrm{mm}$-wavelengths has been shown. The proposed algorithm, already presented in $[1,2]$, has been used in practice to reach a particular conformal serrated edge configuration. Amplitude as well as field phase information of the quiet zone has been presented.

Future theoretical work consists on the development of

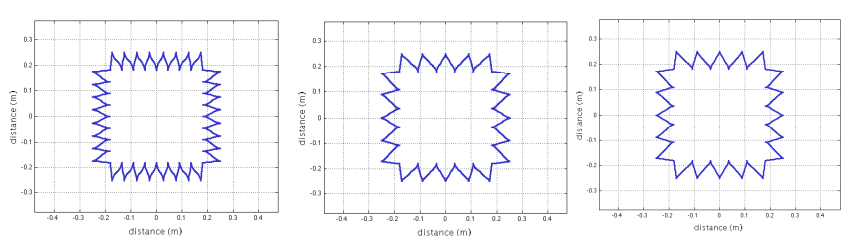

Fig. 4. Minimum ripple reflectors. (a) Amplitude, (b) phase (c) design option
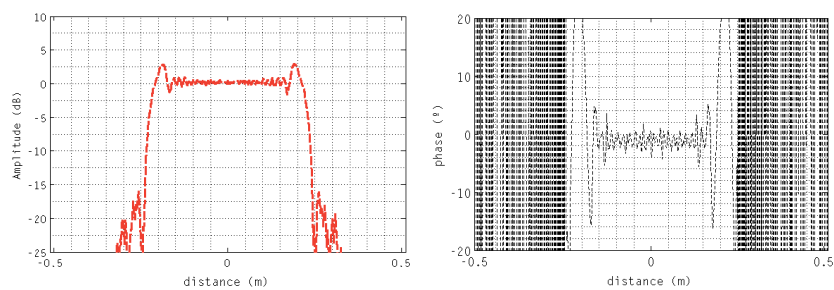

Fig. 5. Amplitude (a) and phase (b); horizontal cuts for the optimum reflector

quiet zone correction techniques [4]. Some work in the field of error clauses modeling-correction has been carried out and is now being included as a piece of the algorithm.

Project development tasks deal with the construction of the reflector, its location inside an anechoic environment and the integration with a millimeter-wave instrumentation subsystem.

\section{ACKNOWLEDGMENT}

This work has been carried out thanks to a Spanish Government FPI grant for Ph.D. Students. The authors also wish to thank the Crocante (TEC2008-06736-C03-01/TEC) and Terasense (CSD2008-00068) projects for the support provided.

\section{REFERENCES}

[1] A. Muñoz-Acevedo, M. Sierra-Castañer, J.L. Besada, "An Efficient Hybrid GO-PWS Algorithm to Analyze Conformal Serrated-Edge Reflectors for Millimeter-Wave Compact Range", IEEE Transactions on Antennas and Propagation, sent for review

[2] A. Muñoz-Acevedo, M. Sierra-Castañer, J.L. Besada, "Antenna Measurement System at $300 \mathrm{GHz}$ for the Terasense Project", $4^{\text {th }}$ European Conference on Antennas and Propagation, Barcelona April $12^{\text {th }}-16^{\text {th }} 2010$

[3] Agilent Technologies 85325A R/Q/U/V/W Millimeter Subsystem: Operating and Service Manual. Available online: http://cp.literature.agilent.com/litweb/pdf/85325-90061.pdf

[4] Joy, E. B, "Antenna Measurement Range Characterization and Compensation", $4^{\text {th }}$ European Conference on Antennas and Propagation, Barcelona April 12 $2^{\text {th }}-16^{\text {th }} 2010$.

[5] P. A. Clemmow, The Plane Wave Spectrum Representation of Electromagnetic Fields, IEEE Press, New jersey, Rissued Edition 1996.

[6] E. B. Joy, R. E. Wilson, "Shaped Edge Serrations for Improved Compact Range Performance," Proc. of the 1987 Antenna Measurement Techniques Association Meeting, Ottawa, Canada, 23-25 September 1986.

[7] Parini, G.; Philippakis, M.; "Use of quiet zone prediction in the design of compact antenna test ranges," IEE Proceedings on Microwaves, Antennas and Propagation, vol.143, no.3, pp.193-199, Jun 1996

[8] Teh-Hong Lee; Burnside, W.D.; , "Performance trade-off between serrated edge and blended rolled edge compact range reflectors," IEEE Transactions on Antennas and Propagation, vol.44, no.1, pp.87-96, Jan 1996 